\title{
Measuring Magnetic Fields Near and Far with the SKA via the Zeeman Effect
}

Timothy Robishaw*1 James A. Green ${ }^{2}$, Gabriele Surcis ${ }^{3}$, Wouter Vlemmings ${ }^{4}$, A. M. S. Richards ${ }^{5}$, Sandra Etoka ${ }^{6}$, Tyler L. Bourke ${ }^{2}$, Vincent Fish ${ }^{7}$, Malcolm Gray ${ }^{5}$, Hiroshi Imai ${ }^{8}$, Busaba Kramer ${ }^{9}$, James McBride ${ }^{10}$, Emmanuel Momjian ${ }^{11}$, Anuj Sarma $^{12}$, and Albert Zijlstra ${ }^{5}$

${ }^{1}$ National Research Council Canada; ${ }^{2}$ SKA Organization; ${ }^{3}$ Joint Institute for VLBI in Europe;

${ }^{4}$ Chalmers University of Technology ${ }^{5}$ Jodrell Bank Centre for Astrophysics; ${ }^{6}$ Hamburger

Sternwarte; ${ }^{7}$ MIT Haystack Observatory; ${ }^{8}$ Kagoshima University; ${ }^{9}$ Max Planck Institute for

Radio Astronomy; ${ }^{10}$ University of California at Berkeley; ${ }^{11}$ National Radio Astronomy

Observatory; ${ }^{12}$ DePaul University;

E-mail: tim.robishaw@nrc-cnrc.gc.ca

The measurement of Zeeman splitting in spectral lines-both in emission and absorption-can provide direct estimates of the magnetic field strength and direction in atomic and molecular clouds, both in our own Milky Way and in external galaxies. This method will probe the magnetic field in the warm and cold neutral components of the interstellar medium, providing a complement to the extensive SKA Faraday studies planning to probe the field in the ionized components.

Advancing Astrophysics with the Square Kilometre Array

June 8-13, 2014

Giardini Naxos, Sicily, Italy

* Speaker.

$\dagger$ On behalf of the SKA Cosmic Magnetism Working Group. 


\section{Introduction}

The Zeeman effect leaves its fingerprint on the circular polarization of a spectral line. A magnetic field threading the gas where a spectral line for certain atomic and molecular transitions is emitted or absorbed will cause the two senses of circular polarization to split apart in frequency. The amplitude of the splitting between the two components is directly proportional to the strength of the magnetic field and a constant that depends on the atomic or molecular transition:

$$
\Delta v[\mathrm{~Hz}]=b B_{\mathrm{los}}[\mu \mathrm{G}](1+z)^{-1},
$$

where $b$ is the Zeeman coefficient of the transition being observed and is measured in $\mathrm{Hz}_{\mu} \mathrm{G}^{-1}$, $B_{\text {los }}$ is the line-of-sight magnetic field measured in microgauss, and $z$ is the redshift of the source. Tables of all known Zeeman coefficients can be found in Heiles et al. (1993) and Robishaw (2008).

The amplitude of the effect measured in the Stokes $V$ spectrum (defined as IEEE right-hand minus left-hand circular polarization) towards sources in the Galactic interstellar medium (ISM) is generally very weak compared to the total intensity of the line measured in the Stokes $I$ spectrum because the splitting is usually very small compared to the width of the line. However, some Galactic maser emission lines can be so narrow that the two circularly polarized components appear completely split; in this case, the splitting is proportional to the total field strength rather than the line-of-sight field (Crutcher et al. 1993).

In this chapter we discuss the potential for using the SKA to measure the Zeeman effect in various targets of interest in increasing order of distance.

\subsection{Assumptions in This Chapter}

The document "SKA1 Imaging Science Performance" (Braun 2014) provides parametrizations for sensitivity in spectral line and continuum modes that are the basis for the performance estimates in this chapter. In particular we utilize their Figure 5, which shows the spectral line sensitivity distribution for a single deep pointing with channel width of $30 \mathrm{~km} \mathrm{~s}^{-1}$ and an integration time of $1000 \mathrm{~h}$. The $L$-band $1-2 \mathrm{GHz}$ range shows that for beam widths between $0.3^{\prime \prime}$ and $300^{\prime \prime}$, we would measure a maximum rms of $12 \mu \mathrm{Jy}$, with a minimum rms of $4 \mu \mathrm{Jy}$ at $10^{\prime \prime}$.

\section{Molecular Clouds and Star Forming Regions in the Milky Way}

The role of magnetic fields during the formation of high-mass stars $\left(M>8 M_{\odot}\right)$ is still a matter of debate, even in the face of many recent investigations, both theoretical and observational (Seifried et al. 2012; Surcis et al. 2013). Recent magnetohydrodynamical simulations show that the collimation of outflows and the formation of accretion discs strongly depend on the strength of magnetic fields (Seifried et al. 2012). In addition, simulations show that magnetic fields also prevent fragmentation, reduce angular momentum, and determine the size of H II regions (Peters et al. 2011; Hennebelle et al. 2011; Seifried et al. 2012). The simulations take into account only relative values of magnetic field strengths obtained considering ratios of magnetic energy with other types of energies (e.g., gravitational energy). Therefore, no real values of magnetic field strengths are currently used in the theoretical calculations. To provide observational measurements 
of magnetic field strengths close to the massive young stellar objects (YSOs) is of great importance. The best probes of magnetic fields at small scales close to YSOs are masers, in particular the Class II methanol maser transition at $6.7 \mathrm{GHz}$ and $22 \mathrm{GHz}$ water masers (Vlemmings et al. 2006a; Surcis et al. 2011, 2013, 2014). Methanol masers, which are the most numerous high-mass star formation (HMSF) maser species, are playing a crucial role in determining the magnetic field morphology (Vlemmings et al. 2010; Surcis et al. 2012, 2013). Moreover, Vlemmings et al. (2006b, 2011) showed that at arcsecond resolution, methanol masers also display significant circular polarization. In fact, $70 \%$ of their total Effelsberg+Parkes flux-limited ( $>50 \mathrm{Jy}$ ) sample (51 sources) show circularly polarized emission. This has also been confirmed at milliarcsecond scale via interferometric observations for a smaller number of sources (Surcis et al. 2012, 2013). However, the exact proportionality between the measured Zeeman-splitting of the methanol maser emission and the magnetic field strength is still under investigation by a team of theoretical chemists and astrophysicists and the final results will be published in 2015. Current instrumentation limits the maser magnetic field studies to up to only two dozen sources, for which only a few lines of sight can be probed. To properly compare with models and simulations, one would need several tens of field measurements on the individual maser features of each source. Additionally, to properly sample different evolutionary stages, a total of up to 100 sources needs to be observed in detail. Unfortunately, our ability to increase this sample is completely limited by the currently available instruments, which allow us to measure the very weak circular polarization signal (usually of the order $0.3 \%-0.5 \%$ ) only towards methanol maser sources with flux exceeding $25 \mathrm{Jy}$ in a reasonable observation time. Fortunately, the upcoming SKA1-MID will allow us to investigate a much larger sample of sources. A $30 \mathrm{~min}$ integration time at a resolution of $0.1 \mathrm{~km} \mathrm{~s}^{-1}$-necessary to detect the splitting-will produce an rms of $9 \mathrm{mJy}$ at $6.7 \mathrm{GHz}$. This means that we could make $5 \sigma$ detections down to a circular polarization fraction of at least $0.3 \%$ towards methanol masers with flux $>15$ Jy. Therefore with SKA1-MID we could easily measure the magnetic field strength in hundreds of HMSF regions and consequently enlarge the investigated area of the Milky Way.

The SKA will be invaluable in the study of Galactic masers, such as very high-resolution follow-up of catalogues like the Methanol (and OH) Multibeam Survey (Green et al. 2009). Multiple transitions provide a chronometer with thousand-year resolution for HMSF concentrated in the Galactic plane (Ellingsen 2007; Breen et al. 2010). Southern hemisphere centimeter-wave studies with resolutions of a few hundred milliarcseconds or better are needed to complement ALMA and to map magnetic field structures. At the other end of stellar evolution, we do not really understand the precise mechanisms whereby cool red giants, AGB stars and supergiants lose mass, nor how almost-spherical stars give rise to asymmetric PNe and SNR. The SKA and ALMA will not only map the kinematics and magnetic fields at high resolution with masers, but, for the first time, relate these to thermal lines and dust emission with high precision.

Evolved OH/IR stars commonly exhibit $\mathrm{OH}$ maser emission that probes the outer part of their circumstellar envelopes (CSEs) where ordered magnetic fields of a few milligauss are detectable (Etoka \& Diamond 2010, 2004). Such observations can trace fields even at the proto-planetarynebula stage (Etoka et al. 2009). Though they start their lives as spherically symmetric objects, a large fraction of planetary nebulae are asymmetrical objects. Although the change of symmetry might be linked primarily to binarity, the organized magnetic fields detected at the distance where $\mathrm{OH}$ masers operate in the CSEs seem to indicate the possible role of magnetism in this morpholog- 
ical development. It is anticipated that thousands of such objects can be detected throughout the Galaxy down to a flux limit of 4 mJy with SKA1-SUR in $\sim 140$ hours (Etoka et al. 2015).

\section{The Magnetic Field Structure of the Milky Way}

\subsection{H I Absorptions Lines against Background Continuum Sources}

The magnetic field in the cold neutral medium (CNM) can be probed via the absorption of continuum emission from compact sources at $21 \mathrm{~cm}$. This method was successfully used by Heiles \& Troland $(2004,2005)$ at the Arecibo telescope to make an uprecedented survey of the CNM towards 41 radio-loud sources; 20 magnetic field components were detected throughout the Milky Way. The limiting sensitivity of their survey was $3 \mathrm{mJy}$. For a $10^{\prime \prime}$ beam and $0.5 \mathrm{~km} \mathrm{~s}^{-1}$ channels, SKA1-MID would achieve this sensitivity in 75 minutes. Therefore, a census of the CNM fields throughout the southern sky should be readily achievable. It should be noted that FAST will certainly make great advances in this endeavour in the northern sky before SKA is online. However, it should be said that single-dish $\mathrm{H}$ I absorption spectra are produced by subtracting an off-position spectrum and are therefore inherently inaccurate; the possibility also arises that polarized sidelobes can contaminate Stokes $V$ spectra for weak sources when using this method. These problems are mitigated by interferometric measurements.

\subsection{H I Self-Absorption}

An intriguing group of targets includes positions that show 21-cm self-absorption (Li \& Goldsmith 2003, the "self" prefix distinguishing this case from that of absorption against a background continuum source). Dozens of clouds throughout the Perseus/Taurus molecular complex show H I absorption at velocities for which $\mathrm{OH}$ and $\mathrm{CO}$ emission is seen. McClure-Griffiths et al. (2006) detected H I self-absorption throughout the Riegel-Crutcher cloud, an $8^{\circ}$-by- $8^{\circ}$ region at the Galactic Center showing an incredible network of long, cold filaments. When the method of Chandrasekhar \& Fermi (1953) is used to investigate stellar polarization measurements along these filaments, magnetic fields in excess of $30 \mu \mathrm{G}$ are inferred. The $\mathrm{H} \mathrm{I}$ absorption profile has a height of $100 \mathrm{~K}$ and a width of $3.5 \mathrm{~km} \mathrm{~s}^{-1}$. A $30 \mu \mathrm{G}$ field produces a Stokes $V$ profile with peak-to-peak extent only $1.6 \%$ of the line height, or $1.6 \mathrm{~K}$. This can be fitted if observed with a sensitivity of $0.3 \mathrm{~K}$. The width of the filaments is between $2^{\prime}-5^{\prime}$, so an integration time of 2 hours per pointing would be required if observed by SKA1-MID with a $120^{\prime \prime}$ beam. The map would require hundreds of pointings, meaning this is best left for SKA2 when the sensitivity will increase tenfold and the field of view will increase twentyfold.

\subsection{Diffuse 21-cm Emission}

Diffuse 21-cm emission is seen in every direction in the sky. This fact, combined with the strong splitting coefficient for the $21-\mathrm{cm}$ line, makes $\mathrm{H}$ I emission an enticing target for Zeeman studies. This line of work was pursued successfully using single dishes for decades before being abandoned in the mid-1990s (Heiles \& Troland 1982; Heiles 1988, 1989, 1996, 1997; Troland \& Heiles 1982a,b; Verschuur 1989; Goodman \& Heiles 1994; Myers et al. 1995). Reliable measurements require a careful accounting of instrumental circular polarization contributions from polarized sidelobes of the telescope. 
As far as we know, nobody has successfully used an interferometer to measure 21-cm Zeeman splitting in diffuse emission. This scenario maintains at the VLA because its circular polarization response contains a severe squint induced by placement of the $L$-band feed far off the symmetry axis of each dish. This is not impossible to model, measure, and account for, but it is a major undertaking. The SKA dishes will be designed such that the secondary and feed alignment satisfy the "Mizigutch criterion" (Mizugutch et al. 1976), which should minimize cross polarization and, therefore, the Stokes $V$ squint. If the circularly polarized beam can be characterized for the SKA, then we presume that the instrumental contribution to a Stokes $V$ measurement can be modeled and accounted for. (Indeed, Agudo et al. 2015 require circular polarization precision of $0.01 \%$ to measure relativistic jet properties.) The many polarization projects that will be undertaken with the SKA guarantee that a thorough accounting of the polarizaton properties of the array will be made, thus enhancing the possibility that the SKA might be the first interferometer to study the Zeeman effect in diffuse 21 -cm emission.

\subsection{OH Masers Tracing the Galactic Magnetic Field}

Davies (1974) found the amazing result that the magnetic field measured in $\mathrm{OH}$ masers seemingly traces the large-scale field that the masers are embedded in. VLBI observations of OH masers at the highest resolutions have demonstrated that maser Zeeman splitting shows field directions and magnitudes which are largely coherent (e.g. Fish et al. 2003; Vlemmings \& van Langevelde 2007). The splitting is often replicated in the lower resolution single-dish studies (Fish et al. 2005; Szymczak \& Gérard 2009). Following the work of Davies (1974), several authors have investigated the concept of maser Zeeman splitting tracing the Galactic magnetic field (e.g. Reid \& Silverstein 1990; Fish et al. 2003; Han \& Zhang 2007), finding fields consistent across kiloparsec scales. These studies were conducted mostly with samples of masers collated from a range of heterogeneous observations; the largest set of systematic observations were those of Fish et al. (2003), but these were limited to only 40 star-forming regions, all visible from the northern hemisphere, and with only a few masers per spiral arm. Despite these limitations, there is an implication that the magnetic fields traced by the masers are tied to the large-scale Galactic magnetic fields, such as those traced by rotation measures (e.g. Han et al. 2006; Brown et al. 2007; Van Eck et al. 2011).

The 'MAGMO' survey (Green et al. 2014) was launched at the Australia Telescope Compact Array (ATCA) in order to potentially map out the magnetic field in the Galactic plane by searching for Zeeman splitting in $\mathrm{OH}$ masers. MAGMO observations achieved a sensitivity of $50 \mathrm{mJy}$ in 30 min of ATCA observing with an 8 " beam, and consisted of targeted follow-up of positions where 6.7 GHz methanol masers were detected. This species of maser is an exclusive tracer of HMSF (Minier et al. 2003; Pestalozzi et al. 2005; Xu et al. 2008; Breen et al. 2013) and as such traces the key structural features of the Galaxy-the spiral arms, 3-kpc arms and bar interaction. The combination of structure and magnetic field information can be a very powerful tool for understanding the dynamics and evolution of the Milky Way.

The logical extension of the MAGMO project would be a blind gridded survey for $\mathrm{OH}$ masers in the Galactic plane using SKA1-MID. A survey covering $-2^{\circ}<b<+2^{\circ}$ and $190^{\circ}<l<60^{\circ}$ would subtend $920 \mathrm{sq}$ deg, providing a sensitivity of $3.7 \mathrm{mJy}$ in one month of observing at the $18 \mathrm{~cm} \mathrm{OH}$ transitions with $0.1 \mathrm{~km} \mathrm{~s}^{-1}$ resolution and a $10^{\prime \prime}$ beam. The scale height for HMSF is well known and this latitude range will capture the vast majority of star forming sources $(>95 \%)$. 
A blind survey to lower sensitivities than achieved by the targeted MAGMO study will bring about a larger detection rate towards regions of HMSF and will increase the number of Zeeman detections. This will markedly improve statistics, both within individual regions, and within Galactic structures such as individual spiral arms. The increased number of measurements will allow for a comparison of maser field directions with those probed via Faraday rotation of polarized continuum sources through nearby sightlines that contain a magnetoionic medium, which will in turn be vastly improved in the SKA era through all-sky polarimetric surveys (Johnston-Hollitt 2015).

An SKA-MAGMO study will explore whether the additional sensitivity that the SKA affords will result in a larger detection rate towards regions of HMSF or a larger number of sources per HMSF site. With the extra sensitivity we would expect an increase in the number of Zeeman pairs/triplets, which will provide more measurements to compare with Faraday rotation measures, decreasing the statistical error. The additional measurements will allow exploration of whether there is any relation to magnetic field strength for much weaker sources, also exploring the proportion of Zeeman pairs compared to Zeeman triplets for weaker sources. The SKA-MAGMO study would further enhance our understanding of the dynamics and evolution of our Galaxy through association with precise positions of HMSF regions (Green et al. 2015).

\section{Zeeman Splitting in External Galaxies}

Masers have the highest luminosity per unit frequency of any radio source, so it is natural to use Zeeman splitting in these beacons to measure magnetic fields in distant galaxies. Luckily, as we saw above in discussing the MAGMO project, $\mathrm{OH}$ has a very large Zeeman coefficient and is therefore a sensitive tracer of magnetic fields. We discuss the possibilities of using $\mathrm{OH}$ masers and megamasers as extragalactic magnetometers.

\subsection{OH Masers in Nearby Galaxies}

SKA2 could be used to survey the local group galaxies for $\mathrm{OH}$ maser emission and Zeeman splitting. If detected, the field structure inferred would be of great interest when comparing to MAGMO results in our own Galaxy because our Galactic study will be confined only to the plane.

Brooks \& Whiteoak (1997) detected OH masers in the LMC, needing a sensitivity of $\sim 40 \mathrm{mJy}$ to obtain a $5 \sigma$ detection in Stokes $I$. For a beam of $10^{\prime \prime}$ and a channel resolution of $0.1 \mathrm{~km} \mathrm{~s}^{-1}$, the SKA1-MID would require less than a minute to detect Zeeman splitting in such a source. A survey of the $\mathrm{OH}$ maser emission in the LMC and SMC is therefore of great interest: any field structure detected in the $\mathrm{OH}$ maser distribution could be compared directly with the fields already mapped in the SMC and LMC via Faraday rotation (Mao et al. 2008; Gaensler et al. 2005).

$\mathrm{OH}$ masers have yet to be detected in M31. Willett (2011) conducted a search using the VLA and found nothing above a $5 \sigma 10 \mathrm{mJy}$ limit concluding that an order of magnitude increase in sensitivity would be required to probe the $\mathrm{OH}$ masers. This would require 10 hours of SKA1-MID integration time with a velocity resolution of $1 \mathrm{~km} \mathrm{~s}^{-1}$.

M82 is the canonical starburst galaxy, located at a distance of 3.5 Mpc. Argo et al. (2010) used 61 hours of VLA observing with a 1" beam to show that OH masers (actually, "kilomasers") are seen throughout M82 with line widths of $\sim 10 \mathrm{~km} \mathrm{~s}^{-1}$ and fluxes as weak as $2 \mathrm{mJy}$. For a $5 \sigma$ detection of all the Stokes $I$ features in M82, we'd require 7 hours of SKA1-MID observing time 
with $1 \mathrm{~km} \mathrm{~s}^{-1}$ channels. However, unlike in the Milky Way, the $\mathrm{OH}$ maser lines in M82 are broad enough that they are only partially split even for large fields of $3 \mathrm{mG}$. The peak-to-peak amplitude of the Zeeman feature in Stokes $V$ will be $50 \%$ of the line height for a splitting induced by a $3 \mathrm{mG}$ field. For the weakest detected $\mathrm{OH}$ maser, we can fit a Zeeman profile easily to a sensitivity limit of $0.3 \mathrm{mJy}$. So to detect Zeeman splitting in all observed maser features using SKA1-MID would require 13 hours of integration. This would allow for a complete mapping of the field as traced by $\mathrm{OH}$ masers in M82.

\subsection{Megamasers}

Megamasers have been detected in hundreds of galaxies at $z \sim 0.1$, and, with the aid of lensing, as far away as $z=2.6$ (Castangia et al. 2011). Intrinsically compact both spatially (detectable down to micro-arcsec scales) and spectrally (sub-km s${ }^{-1}$ ), they provide the best directly mappable tracers of high-resolution structure in the inner few hundred parsecs of active galaxies. The early stages of the SKA are suited to studies of the larger-scale $\mathrm{OH}$ masers (rest frequency $1.67 \mathrm{GHz}$ ) associated with nuclear starbursts and Seyfert galaxies. The position of individual maser spots can be measured with an accuracy proportional to (beam size)/(signal-to-noise). For example, at a redshift of order 0.05, MERLIN (resolution 120 mas) could resolve 10-parsec details in Markarian 231 and 273 (Richards et al. 2005; Yates et al. 2000) showing warped discs and orbiting mass densities of 300-900 $\mathrm{M}_{\odot} \mathrm{pc}^{-3}$.

The most exciting use of $\mathrm{OH}$ megamasers (OHMs) has been the measurement of in situ magnetic fields in 15 external galaxies by Robishaw et al. (2008) and McBride \& Heiles (2013), who used the high spectral resolution of Arecibo to separate multiple Zeeman components and compared these with VLBI and MERLIN total-intensity lower-resolution spectra extracted from spatially-discrete regions. The inferred magnetic field strengths of $0.5-80 \mathrm{mG}$ provide an energy density comparable to the hydrostatic gas pressure in the masing regions, likely to be active star formation sites. The results from OHMs also suggest that magnetic fields are dynamically important throughout the central starburst region, whereas weaker magnetic fields are inferred from radio synchrotron measurements assuming equipartition. The OHM-derived estimates are consistent with the linearity of the far-infrared-radio correlation (McBride et al. 2014).

The total OHM velocity span can exceed $1000 \mathrm{~km} \mathrm{~s}^{-1}$ but typical Zeeman splitting detections have been associated with lines that have velocity widths $<20 \mathrm{~km} \mathrm{~s}^{-1}$ and flux densities $>3 \mathrm{mJy}$. The Arecibo detections required an rms flux density of $3 \mathrm{mJy}$ in $0.5 \mathrm{~km} \mathrm{~s}^{-1}$ channels. SKA1-MID will be able to survey all southern galaxies for OHM Zeeman splitting; a 1" beam at $1.6 \mathrm{GHz}$ will reach $1 \mathrm{mJy}$ sensitivity in 2 hours with $0.5 \mathrm{~km} \mathrm{~s}^{-1}$ channels, sufficient to detect analogues of the sources in the Arecibo sky. If all SKA-visible Arecibo targets were searched with this threefold improvement in sensitivity, we could expect to double the number of galaxies with Zeeman detections in 2 days of observing with SKA1-MID. The southern sky has not been thoroughly searched for OHMs; blind 21-cm surveys with the SKA are bound to catalog the southern OHM population. Deep follow-up observations of the southern OHM galaxies will yield at least another doubling of the number of Zeeman-detected galaxies; sampling the 100 brightest OHMs would require 200 hours of SKA1-MID time. The most distant Zeeman detections occurred in OHMs at $z \sim 0.2$. The sensitivity increase afforded by SKA2 will allow Zeeman splitting to be probed in OHMs out 
past $z=1$, allowing these beacons to become invaluable tools for studying galactic magnetic fields through cosmic time.

Previous OHM studies have relied on literature VLBI imaging, but this adds uncertainty as the intervals between observations are comparable to the variability timescales of compact masing clouds. SKA2 will reach the same $1 \mathrm{mJy}$ rms per 120 mas beam in the same time in $25 \mathrm{~km} \mathrm{~s}^{-1}$ channels, allowing comparison between simultaneous high spectral- and high spatial-resolution results. SKA2 will extend its reach to more highly redshifted $\mathrm{OH}$ masers and possibly to $22 \mathrm{GHz}$ water masers, which trace material orbiting black holes and jet-ISM interactions on sub-pc scales. However, the splitting coefficient for $\mathrm{H}_{2} \mathrm{O}$ is 1000 times weaker than that for $\mathrm{OH}$ such that Zeeman splitting in water megamasers has thus far eluded detection (Modjaz et al. 2005).

\subsection{H I Absorption in Damped Ly $\alpha$ Absorbers}

Damped Ly $\alpha$ absorbing systems (DLAs) are a class of quasar absorber in which hydrogen remains mostly neutral. The neutral gas content of the Universe is dominated up to redshift 5 by DLAs and the $\mathrm{H}$ I layers producing the absorption are considered to be the progenitors of modern galaxies. DLAs are perhaps the best and only sample of an interstellar medium in the high-redshift Universe (Wolfe et al. 2005). As such, the possibility of measuring Zeeman splitting in the 21 $\mathrm{cm}$ line absorption in these systems would allow us to test the importance of the role of magnetic fields in galaxy formation and evolution and constrain dynamo models for the generation and amplification of magnetic fields in the early Universe. Wolfe et al. $(2011,2008)$ describe Green Bank Telescope observations of a DLA at $z=0.692$ towards 3C 286. No Zeeman signature was detected in the Stokes $V$ spectrum at $839.40 \mathrm{MHz}$ down to a field limit of $17 \mu \mathrm{G}$. It would take SKA1-MID 90 min to probe down to $5 \mu \mathrm{G}$ field strengths in this DLA with $1.5 \mathrm{~km} \mathrm{~s}^{-1}$ channels. The ability to probe such systems to lower sensitivities and therefore lower limiting magnetic fields is tantalizing. Because of the large redshifts of these systems, all observations would be carried out in bands 1 and 2. These DLAs are viable targets for all phases of SKA deployment.

\section{Conclusions}

Measurement of the Zeeman effect is sensitivity limited. Our ability to use this method to probe magnetic fields in the Milky Way and beyond is completely dependent on the development of telescopes with better sensitivity than is currently achievable by the world's largest observatories. The SKA will make significant improvements in our pursuit of measuring Zeeman splitting in spectral lines, both in emission and absorption. These measurements will provide direct estimates of and upper limits to the magnetic field strength and direction in atomic and molecular clouds, both in our own Milky Way and in external galaxies. This method will probe the magnetic field in the warm and cold neutral components of the interstellar medium, providing a complement to the extensive SKA Faraday studies planning to probe the field in the ionized components.

The early phase SKA1 is estimated to yield a $50 \%$ reduction in sensitivity, resulting in a quadrupling of the the required integration times calculated here. Of the possible SKA1 experiments described, only the $\mathrm{OH}$ megamaser survey would be prohibitively long and require the full deployment of SKA1. 


\section{References}

Agudo, I., Böttcher, M., Falcke, H., et al. 2015, "Studies of Relativistic Jets in Active Galactic Nuclei with SKA", in proc. Advancing Astrophysics with the Square Kilometre Array, PoS(AASKA14)093

Argo, M. K., Pedlar, A., Beswick, R. J., Muxlow, T. W. B., \& Fenech, D. M. 2010, MNRAS, 402, 2703

Braun, R. 2014, "SKA1 Imaging Science Performance", Document SKA-TEL-SKO-DD-XXX Rev. A Draft 5

Breen, S. L., Ellingsen, S. P., Caswell, J. L., \& Lewis, B. E. 2010, MNRAS, 401, 2219

Breen, S. L., Ellingsen, S. P., Contreras, Y., et al. 2013, MNRAS, 435, 524

Brooks, K. J., \& Whiteoak, J. B. 1997, MNRAS, 291, 395

Brown, J. C., Haverkorn, M., Gaensler, B. M., et al. 2007, ApJ, 663, 258

Castangia, P., Impellizzeri, C. M. V., McKean, J. P., et al. 2011, A\&A, 529, A150

Chandrasekhar, S., \& Fermi, E. 1953, ApJ, 118, 113

Crutcher, R. M., Troland, T. H., Goodman, A. A., et al. 1993, ApJ, 407, 175

Davies, R. D. 1974, in IAU Symp. 60, Galactic Radio Astronomy, ed. F. J. Kerr \& S. C. Simonson (Dordrecht: Reidel), 275

Ellingsen, S. P. 2007, MNRAS, 377, 571

Etoka, S., \& Diamond, P. 2004, MNRAS, 348, 34

Etoka, S., \& Diamond, P. J. 2010, MNRAS, 406, 2218

Etoka, S., Engels, D., Imai, H., et al. 2015, "OH masers in the Milky Way and Local Group galaxies in the SKA era", in proc. Advancing Astrophysics with the Square Kilometre Array, PoS(AASKA14) 125

Etoka, S., Zijlstra, A., Richards, A. M., Matsuura, M., \& Lagadec, E. 2009, in Astronomical Society of the Pacific Conference Series 404, The Eighth Pacific Rim Conference on Stellar Astrophysics: A Tribute to Kam-Ching Leung, ed. B. Soonthornthum, S. Komonjinda, K. S. Cheng, \& K. C. Leung (San Francisco: ASP), 311

Fish, V. L., Reid, M. J., Argon, A. L., \& Menten, K. M. 2003, ApJ, 596, 328

Fish, V. L., Reid, M. J., Argon, A. L., \& Zheng, X.-W. 2005, ApJS, 160, 220

Gaensler, B. M., Haverkorn, M., Staveley-Smith, L., et al. 2005, Science, 307, 1610

Goodman, A. A., \& Heiles, C. 1994, ApJ, 424, 208

Green, J. A., Caswell, J. L., Fuller, G. A., et al. 2009, MNRAS, 392, 783

Green, J. A., Gray, M. D., Robishaw, T., Caswell, J. L., \& McClure-Griffiths, N. M. 2014, MNRAS, 440, 2988

Green, J. A., van Langevelde, H. J., Brunthaler, A., et al. 2015, "Maser Astrometry with the SKA", in proc. Advancing Astrophysics with the Square Kilometre Array, PoS(AASKA14)119

Han, J. L., Manchester, R. N., Lyne, A. G., Qiao, G. J., \& van Straten, W. 2006, ApJ, 642, 868

Han, J. L., \& Zhang, J. S. 2007, A\&A, 464, 609

Heiles, C. 1988, ApJ, 324, 321

1989, ApJ, 336, 808

1996, ApJ, 466, 224

1997, ApJS, 111, 245 
Heiles, C., Goodman, A. A., McKee, C. F., \& Zweibel, E. G. 1993, in Protostars and Planets III, ed. E. H. Levy \& J. I. Lunine (Tucson: Univ. Arizona Press), 279

Heiles, C., \& Troland, T. H. 1982, ApJ, 260, L23

- 2004, ApJS, 151, 271

. 2005, ApJ, 624, 773

Hennebelle, P., Commerçon, B., Joos, M., et al. 2011, A\&A, 528, A72

Johnston-Hollitt, M. 2015, "Using SKA Rotation Measures to Reveal the Mysteries of the Magnetised Universe", in proc. Advancing Astrophysics with the Square Kilometre Array, PoS(AASKA14)092

Li, D., \& Goldsmith, P. F. 2003, ApJ, 585, 823

Mao, S. A., Gaensler, B. M., Stanimirović, S., et al. 2008, ApJ, 688, 1029

McBride, J., \& Heiles, C. 2013, ApJ, 763, 8

McBride, J., Quataert, E., Heiles, C., \& Bauermeister, A. 2014, ApJ, 780, 182

McClure-Griffiths, N. M., Dickey, J. M., Gaensler, B. M., Green, A. J., \& Haverkorn, M. 2006, ApJ, 652, 1339

Minier, V., Ellingsen, S. P., Norris, R. P., \& Booth, R. S. 2003, A\&A, 403, 1095

Mizugutch, Y., Akagawa, M., \& Yokoi, H. 1976, ISA Proceedings, 2

Modjaz, M., Moran, J. M., Kondratko, P. T., \& Greenhill, L. J. 2005, ApJ, 626, 104

Myers, P. C., Goodman, A. A., Gusten, R., \& Heiles, C. 1995, ApJ, 442, 177

Pestalozzi, M. R., Minier, V., \& Booth, R. S. 2005, A\&A, 432, 737

Peters, T., Banerjee, R., Klessen, R. S., \& Mac Low, M.-M. 2011, ApJ, 729, 72

Reid, M. J., \& Silverstein, E. M. 1990, ApJ, 361, 483

Richards, A. M. S., Knapen, J. H., Yates, J. A., et al. 2005, MNRAS, 364, 353

Robishaw, T. 2008, Ph.D. thesis, University of California at Berkeley

Robishaw, T., Quataert, E., \& Heiles, C. 2008, ApJ, 680, 981

Seifried, D., Banerjee, R., Pudritz, R. E., \& Klessen, R. S. 2012, MNRAS, 423, L40

Surcis, G., Vlemmings, W. H. T., Curiel, S., et al. 2011, A\&A, 527, A48

Surcis, G., Vlemmings, W. H. T., van Langevelde, H. J., et al. 2014, A\&A, 565, L8

Surcis, G., Vlemmings, W. H. T., van Langevelde, H. J., \& Hutawarakorn Kramer, B. 2012, A\&A, 541, A47

Surcis, G., Vlemmings, W. H. T., van Langevelde, H. J., Hutawarakorn Kramer, B., \& QuirogaNuñez, L. H. 2013, A\&A, 556, A73

Szymczak, M., \& Gérard, E. 2009, A\&A, 494, 117

Troland, T. H., \& Heiles, C. 1982a, ApJ, 252, 179

- 1982b, ApJ, 260, L19

Van Eck, C. L., Brown, J. C., Stil, J. M., et al. 2011, ApJ, 728, 97

Verschuur, G. L. 1989, ApJ, 339, 163

Vlemmings, W. H. T., Diamond, P. J., van Langevelde, H. J., \& Torrelles, J. M. 2006a, A\&A, 448, 597

Vlemmings, W. H. T., Harvey-Smith, L., \& Cohen, R. J. 2006b, MNRAS, 371, L26

Vlemmings, W. H. T., Surcis, G., Torstensson, K. J. E., \& van Langevelde, H. J. 2010, MNRAS, 404, 134

Vlemmings, W. H. T., Torres, R. M., \& Dodson, R. 2011, A\&A, 529, A95 
Vlemmings, W. H. T., \& van Langevelde, H. J. 2007, A\&A, 472, 547

Willett, K. W. 2011, Ph.D. thesis, PhD Thesis, University of Colorado, 2011

Wolfe, A. M., Gawiser, E., \& Prochaska, J. X. 2005, ARA\&A, 43, 861

Wolfe, A. M., Jorgenson, R. A., Robishaw, T., Heiles, C., \& Prochaska, J. X. 2008, Nature, 455, 638 2011, ApJ, 733, 24

Xu, Y., Li, J. J., Hachisuka, K., et al. 2008, A\&A, 485, 729

Yates, J. A., Richards, A. M. S., Wright, M. M., et al. 2000, MNRAS, 317, 28 